\title{
A basic dynamic transmission model of Staphylococcus aureus in the US population
}

\author{
C. HOGEA ${ }^{1 *}$, T. VAN EFFELTERRE ${ }^{2}$ AND C. J. ACOSTA ${ }^{1,3}$ \\ ${ }^{1}$ GlaxoSmithKline Vaccines, King of Prussia, PA, USA \\ ${ }^{2}$ GlaxoSmithKline Vaccines, Wavre, Belgium \\ ${ }^{3}$ Merck, West Point, PA, USA
}

Received 26 October 2012; Final revision 16 April 2013; Accepted 16 April 2013; first published online 23 May 2013

\section{SUMMARY}

We present a basic mathematical model of Staphylococcus aureus transmission in the USA based on natural history of infection and nationally representative data. We employed a Susceptible-Colonized-Infected-Recovered-Susceptible compartmental modelling framework with two different phenotypes of $S$. aureus: methicillin-susceptible (MSSA) and methicillin-resistant (MRSA). The model is dynamic and accounts for the US population growth. For model calibration/validation, we used published 1999-2005 S. aureus infection data in conjunction with the 2001-2004 National Health and Nutrition Examination Survey colonization data. Baseline model projections illustrated how MRSA might continue to expand and gradually replace MSSA over time, in the absence of intervention, if there is strong competition for colonization. The model-based estimate of the basic reproduction number $\left(R_{0}\right)$ highlights the need for infection control. We illustrate the potential population-level impact of intervention with a hypothetical S. aureus vaccination component.

Key words: Mathematical modelling, public health, Staphylococcus aureus, transmission model.

\section{INTRODUCTION}

Staphylococcus aureus is a Gram-positive bacterium, a ubiquitous pathogen that constitutes one of the major causes of infections in humans [1]. The most consistently identified ecological niche for $S$. aureus in humans is in the anterior nares and up to one third of healthy people carry this organism in the nose at any time [2]. Asymptomatic carriage of $S$. aureus often precedes disease, and carriage isolates are frequently identical to strains recovered from subsequent clinical infection [2]. Colonization offers a reservoir

\footnotetext{
* Author for correspondence: Dr C. Hogea, GlaxoSmithKline Vaccines, 2301 Renaissance Blvd, Ste RN0510, King of Prussia, PA, USA.

(Email: cosmina.s.hogea@gsk.com)
}

from where the pathogen can access the bloodstream when breaches appear in host defence systems but infections can occur without nasal colonization [2], particularly in the case of community-associated infections. The main mode of transmission of $S$. aureus is through direct contact (person-person/fomiteperson) $[1,2]$.

Methicillin-resistant S. aureus (MRSA) clones have been recognized as a leading cause of nosocomial infections in the USA and around the world for several decades [3, 4]. However, MRSA is no longer only a nosocomial pathogen and in recent years has emerged as a significant health threat in community settings in people without known risk factors or prior healthcare exposure [5].

Historically, penicillin resistance in $S$. aureus was initially reported in hospital settings $1-2$ years after 
penicillin was introduced in 1941 [6]. A rise in the community rates of penicillin-resistant strains was documented shortly after and in the 1970s the two rates converged and accounted for $70-85 \%$ of strains [6]. Methicillin was introduced in 1961 and reports of resistant strains in hospitals emerged less than 1 year later [6]. The prevalence of MRSA has since progressively increased in hospitals in the USA and subsequently in the community during the past 20 years [6].

National hospitalization and resistance data were used to estimate the annual number of hospitalizations and deaths associated with $S$. aureus and MRSA from 1999 to 2005 [7]. During this period, the estimated number of $S$. aureus-related hospitalizations increased by $62 \%$, and the estimated number of MRSA-related hospitalizations more than doubled [7]. With the prevalence of MRSA infection in the USA increasing over the past decade due to the emergence of community-acquired MRSA (CA-MRSA), there is a need to evaluate better the transmission of $S$. aureus at the population level.

Dynamic transmission models are powerful mathematical tools for simulating disease transmission in a population. Such tools can help test various hypotheses/scenarios, project potential future trends under various assumptions and assess the impact of interventions at the population level. In this context, we developed a mathematical model of $S$. aureus transmission in the US population based on the natural history of infection and nationally representative data. This type of model was used to test working hypotheses to understand and explain observed trends, investigate the potential spread of MRSA and methicillin-susceptible S. aureus (MSSA) in the population, and project potential future trends and related estimates for the burden of infection. A secondary objective was to explore the potential impact of systematic population-level interventions, illustrated here with a hypothetical $S$. aureus vaccination component.

\section{METHODS}

We employed a Susceptible-Colonized-InfectedRecovered-Susceptible (SCIRS) dynamic, populationbased, mechanistic, compartmental modelling framework, with two competing phenotypes of $S$. aureus [8]. The model structure is shown in Figure 1. Corresponding equations for the baseline model are shown in the Supplementary online material.

This basic model version includes both MRSA and MSSA, with the assumption of strong competition for colonization of susceptible hosts, i.e. the average individual can be colonized either with MRSA or with MSSA, but not simultaneously with both for sustained periods of time $[9,10]$. This assumption can be relaxed to allow for an explicit niche of co-existence for both MRSA and MSSA [8], but additional data would be needed to disambiguate (remove uncertainty) at the time of model calibration. For simplicity and in the absence of strong supporting evidence, we did not explicitly account for potential MRSA/ MSSA co-colonization.

Symptomatic MSSA or MRSA infection can occur via two alternative routes: either through primary nasal colonization (with other body sites potentially colonized) followed by infection, or by direct (no apparent/persistent nasal or other body site colonization) infection of susceptible hosts through contact with, for example, colonized or infected persons or contaminated fomites. Susceptible hosts are not yet colonized or infected with MRSA or MSSA, but colonization might occur via adequate contact with other colonized or infected individuals. The concept of a 'Recovered' compartment was introduced here to allow and explore the possibility of a time delay for individuals who had recently recovered from a symptomatic infection before again becoming fully susceptible to colonization or infection, potentially due to shortterm impact of some form of antibiotic treatment.

The proposed model is fully dynamic, accounting for an estimated projected US population growth rate of $1.26 \%$ per year (based on Census data [11]). The simplified model, without age stratification, was calibrated and validated based on published US national infection data (1999-2005) [7] and colonization data (2001-2004) [12], respectively. In Klein et al. [7], the annual number of hospitalized $S$. aureus/ MRSA infections in the USA between 1999 and 2005 was estimated based on national hospitalization and resistance data. A nationally representative survey of colonization with $S$. aureus/MRSA was also conducted from 2001 to 2004 as part of the National Health and Nutrition Examination Survey (NHANES) [12], which is the Centers for Disease Control and Prevention's biennial biomonitoring survey of a large sample of US residents. S. aureus/ MRSA nasal colonization was reported for the periods 2001-2002 and 2003-2004. Data from these two surveys were used to calibrate the model.

A primary aim of the study was to construct a basic mechanistic framework which properly reflects and uses the US national trends reported over time to 


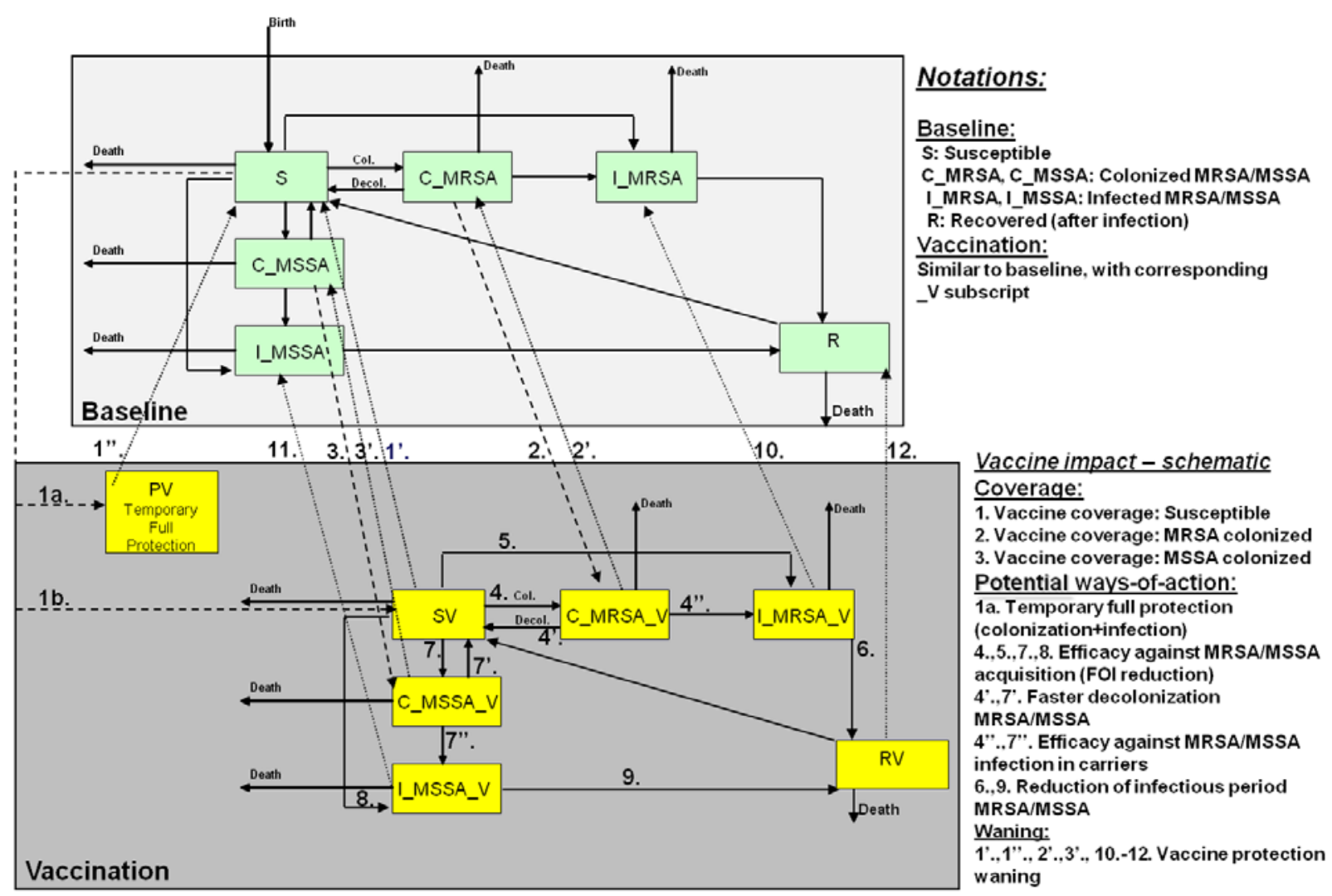

Fig. 1 [colour online]. Schematic of the model structure: baseline and an added vaccination component, respectively. In the baseline model, individuals are born into the susceptible state and can die in any of the model states. Susceptible people can become colonized or directly infected (no persistent colonization) either with MRSA or with MSSA, based on the corresponding component of the force of infection (FOI, i.e. per susceptible risk of infection). A percentage of the individuals colonized with MRSA or MSSA can subsequently develop invasive infection with the same strain. Colonized people naturally clear after a period of time. We allow for the possibility (short-lived) that people recovered from invasive infection might be temporarily protected (e.g. as a consequence of antibiotic treatment) - after which they return to a fully susceptible state. The arrows represent the corresponding flows of individuals between different states. Corresponding mathematical equations (set of nonlinear ordinary differential equations) are given in Supplementary Figure S1. This type of modelling framework can consider a variety of potential vaccine modes of action - illustrated in the schematic here for completeness.

make further potential projections at the national level under various assumptions or working hypotheses. In this framework, all model parameters were regarded as averaged across the entire US population and the model outputs represented total (hospitalized and non-hospitalized combined) prevalence/incidence of MRSA and MSSA infections in the USA at the national level.

Best-fit estimates were obtained by simultaneously minimizing the difference, in the least-square sense, between all data points and the corresponding model outcomes. However, since MRSA infection data were available for hospitalized infections only [7], scaling factors representing the proportion of hospitalizations out of the total infections for MRSA and MSSA, respectively, were introduced to project model-based hospitalizations and compare them against the actual data. These factors were assumed to be constant for simplicity, within ranges of $50-70 \%$ for MRSA and $40-60 \%$ for MSSA (combining $S$. aureus/MRSA infection data reported in [13] for both inpatient and outpatient settings), and further included in the overall parameter estimation to yield corresponding optimal values.

The estimation of model parameters can be formulated as a sophisticated constrained optimization problem. To enforce biological and epidemiological plausibility, whenever available, additional information was incorporated in the constraints (e.g. feasible/plausible parameter ranges) $[14,15]$. Additional details are provided in the Supplementary material. The national datasets for infection and colonization 
Table 1. Best-fit estimates for the baseline model parameters

\begin{tabular}{|c|c|c|c|}
\hline $\begin{array}{l}\text { Parameter } \\
\text { number }\end{array}$ & Parameter nomenclature & $\begin{array}{l}\text { Symbol (see } \\
\text { Supplementary material) }\end{array}$ & $\begin{array}{l}\text { Value (unit) (best-fit } \\
\text { model estimate) }\end{array}$ \\
\hline \multicolumn{4}{|c|}{ Transmission rates } \\
\hline 1 & Colonized by colonized MRSA & $\lambda_{1}^{\mathrm{C}}$ & $1.46 \times 10^{-2}\left(\mathrm{day}^{-1}\right)$ \\
\hline 2 & Colonized by infected MRSA & $\lambda_{1}^{\mathrm{I}}$ & $1 \cdot 76 \times 10^{-2}\left(\mathrm{day}^{-1}\right)$ \\
\hline 3 & Infected by colonized MRSA & $\beta_{1}^{\mathrm{C}}$ & $7 \cdot 30 \times 10^{-6}\left(\mathrm{day}^{-1}\right)$ \\
\hline 4 & Infected by infected MRSA & $\beta_{1}^{\mathrm{I}}$ & $1.49 \times 10^{-2}\left(\right.$ day $\left.^{-1}\right)$ \\
\hline 5 & Colonized by colonized MSSA & $\lambda_{2}^{\mathrm{C}}$ & $1.46 \times 10^{-2}\left(\mathrm{day}^{-1}\right)$ \\
\hline 6 & Colonized by infected MSSA & $\lambda_{2}^{\bar{I}}$ & $4.01 \times 10^{-2}\left(\mathrm{day}^{-1}\right)$ \\
\hline 7 & Infected by colonized MSSA & $\beta_{2}^{\mathrm{C}}$ & $6.87 \times 10^{-6}\left(\mathrm{day}^{-1}\right)$ \\
\hline 8 & Infected by infected MSSA & $\beta_{2}^{1}$ & $3.61 \times 10^{-3}\left(\mathrm{day}^{-1}\right)$ \\
\hline \multicolumn{4}{|c|}{ Clearance rates } \\
\hline 9 & Colonized MRSA clearance & $\alpha_{1}$ & $9 \cdot 80 \times 10^{-3}\left(\mathrm{day}^{-1}\right)$ \\
\hline 10 & Colonized MSSA clearance & $\alpha_{2}$ & $1 \cdot 02 \times 10^{-2}\left(\right.$ day $\left.^{-1}\right)$ \\
\hline \multicolumn{4}{|c|}{ Infection rates } \\
\hline 11 & Colonized MRSA progressing to infection & $\gamma_{1}$ & $1 \cdot 20 \times 10^{-4}\left(\mathrm{day}^{-1}\right)$ \\
\hline 12 & Colonized MSSA progressing to infection & $\gamma_{2}$ & $8 \cdot 22 \times 10^{-6}\left(\right.$ day $\left.^{-1}\right)$ \\
\hline \multicolumn{4}{|l|}{ Other } \\
\hline 13 & Mean recovery period for MRSA infections & $\eta_{1}$ & $14 \cdot 60$ (days) \\
\hline 14 & Mean recovery period for MSSA infections & $\eta_{2}$ & $8 \cdot 30$ (days) \\
\hline 15 & Mean temporary protection period & $\mu$ & $7 \cdot 70$ (days) \\
\hline 16 & $\begin{array}{l}\text { Scaling factor MRSA (proportion MRSA infections } \\
\text { hospitalized/total MRSA infections) }\end{array}$ & - & $0 \cdot 58$ \\
\hline 17 & $\begin{array}{l}\text { Scaling factor MSSA (proportion MSSA infections } \\
\text { hospitalized/total MSSA infections) }\end{array}$ & - & $0 \cdot 45$ \\
\hline
\end{tabular}

$[7,12]$ were simultaneously fitted over time to yield the best agreement between model outcomes and corresponding data (Table 1).

For exploratory purposes, we included a hypothetical vaccination component on top of the baseline model, as illustrated in Figure 1. An annual vaccine uptake was assumed as follows: $3 \cdot 5 \%$ of the susceptible population, $5 \%$ of the MRSA-colonized population and $5 \%$ of the MSSA-colonized population. This amounted to a cumulated coverage of about $5 \%$ of the total US population, vaccinated once per year. Regarding potential effects of the vaccine (see Fig. 1), the hypothetical case scenario shown assumed a $40 \%$ reduction in the risk of colonization, $50 \%$ reduction in the risk of direct infection [16] and $40 \%$ reduction in the risk of infection in individuals colonized by MRSA or MSSA.

The purpose here is essentially to illustrate the potential and versatility of this type of modelling framework to capture a broad host of potential vaccine effects, which can play important roles in subsequent analyses attempting to estimate the impact of vaccination. This was already reported for vaccines against other pathogens such as Neisseria meningitidis
[17] or Streptococcus (Str.) pneumoniae [18] that demonstrated efficacy against infection and colonization. In this context, vaccine-related model parameters such as vaccine coverage and different efficacies/risk reductions were assumed to have values between $0 \%$ and $100 \%$. Here we are only attempting to illustrate with a pertinent example. Another important parameter in practice is the mean duration of vaccine protection in vaccinated individuals. For comparative purposes, we assumed a mean duration of vaccine protection of 1 year $v s .5$ years.

All numerical simulations were carried out in MATLAB R2010b (MathWorks, USA).

\section{RESULTS}

The model proved able to capture both the colonization and infection data, with estimated $P$ values for model/data correlation of $<0.0001$ for MRSA infection and $<0.05$ for MSSA infection. In order to test the predictive capabilities of the model, the 2004-2005 MRSA infection data points were omitted at calibration; they were reasonably rendered by the best-fit model. Corresponding best-fit plots are 
(a)

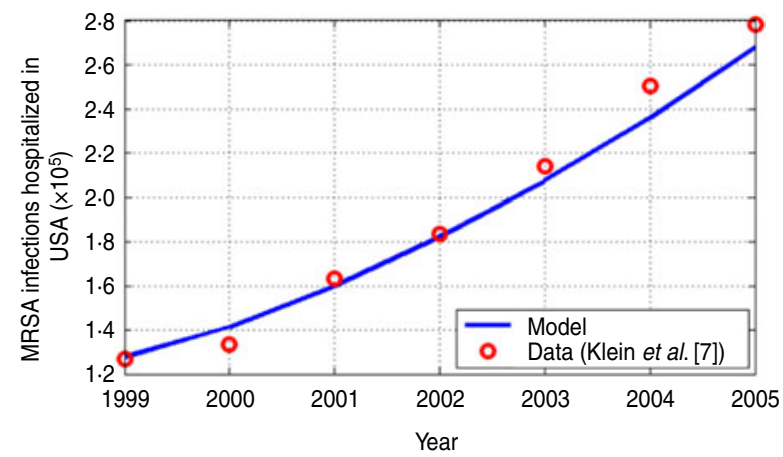

(b)

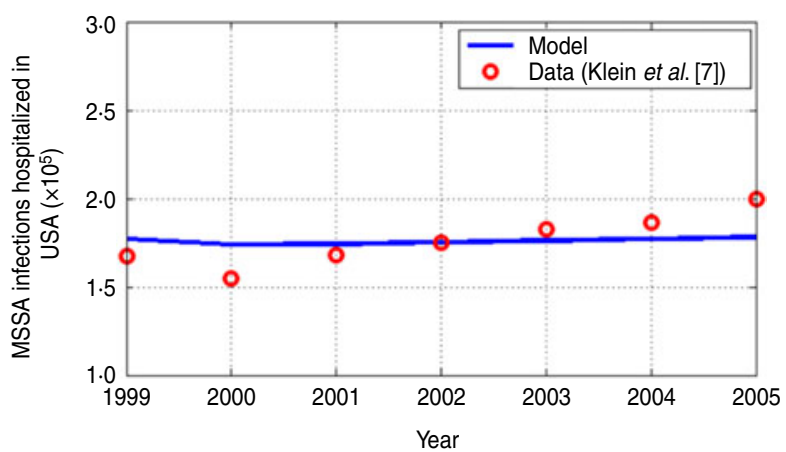

(c)

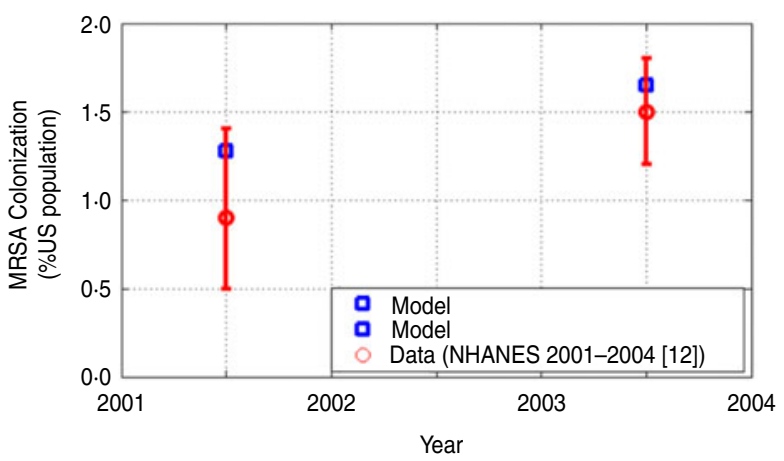

(d)

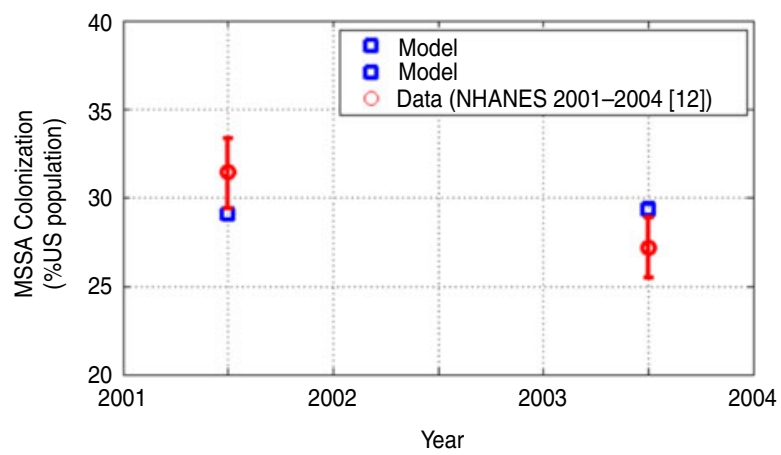

Fig. 2 [colour online]. Best-fit model vs. observed, for MRSA and MSSA infections $(a, b)$ hospitalized and $(c, d)$ colonized. NHANES 2001-2004 biennial colonization data are shown with the 95\% confidence intervals (vertical bars), as published [12]. The 1999-2003 annual numbers of MRSA infections hospitalized [7] were used for model calibration, while the 2004-2005 [7] data points were omitted for validation; the calibrated model succeeded in capturing these data fairly well. (a) Number of MRSA infections hospitalized in he USA 1999-2005: best-fit model calibration against corresponding data in [7]. (b) Number of MSSA infections hospitalized in the USA 1999-2005: best-fit model calibration against corresponding data in [7]. (c) Biennial prevalence of MRSA colonization 2001-2004: best-fit model calibration against corresponding data in [12]. (d) Biennial prevalence of MSSA colonization 2001-2004: best-fit model calibration against corresponding data in [12].

shown in Figure 2 for best-fit model parameter values given in Table 1. With model parameters calibrated based on the national datasets described above, baseline model projections illustrate how MRSA might expand and gradually replace MSSA over time at the US population level, under the assumption of strong MSSA/MRSA competition for colonization.

The model-based estimates of the basic reproduction number $\left(R_{0}\right)$, which quantifies the potential of spread, were $>1$ for both MRSA and MSSA, with an estimated $R_{0}$ for MRSA slightly higher than for MSSA (1.5 vs. 1.4). Using a next-generation matrix method [19], the estimates of $R_{0}$ from the best-fit model are indicative of tight MRSA/MSSA competition. Ultimately, expansion of the strain phenotype with the highest $R_{0}$ (here MRSA) is favoured. However, the model projects a potential gradual replacement of MSSA over a timeframe of several decades (Figs 3 and 4 ). The model also projects a sustained increase over time in the annual number of $S$. aureus infections in the US population, increasingly due to MRSA.

Regarding the possibility of a time delay for individuals who had recently recovered from a symptomatic infection before again becoming fully susceptible to colonization or infection, the best-fit model calibration against data estimated a very short period (7.7 days) of potential temporary protection (Table 1).

In order to assess model output variations to small perturbations in the model parameters, we conducted a parametric sensitivity analysis for the model governing system of ordinary differential equations (ODEs, shown in the Supplementary material) and computed numerically derivatives (sensitivities) for all the model state variables $(n=6)$ with respect to the 17 parameters listed in Table 1, over the timeframe used for the simulations in Figures 3 and 4. Based on this analysis, summarized and illustrated in Supplementary Figure S2, the model outputs are most sensitive to the following 


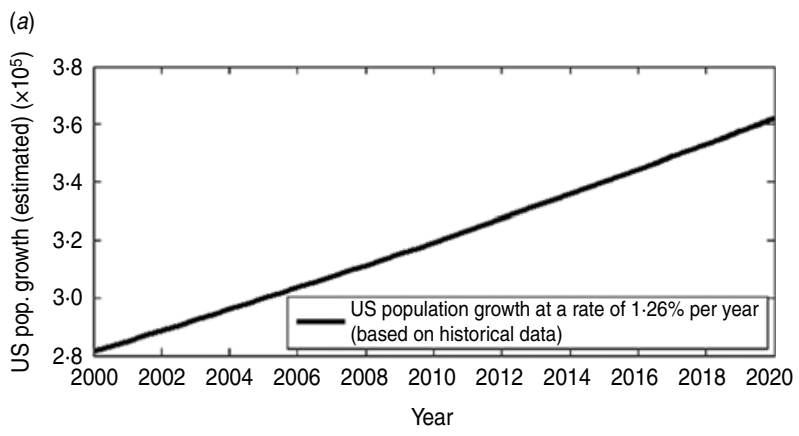

(b)

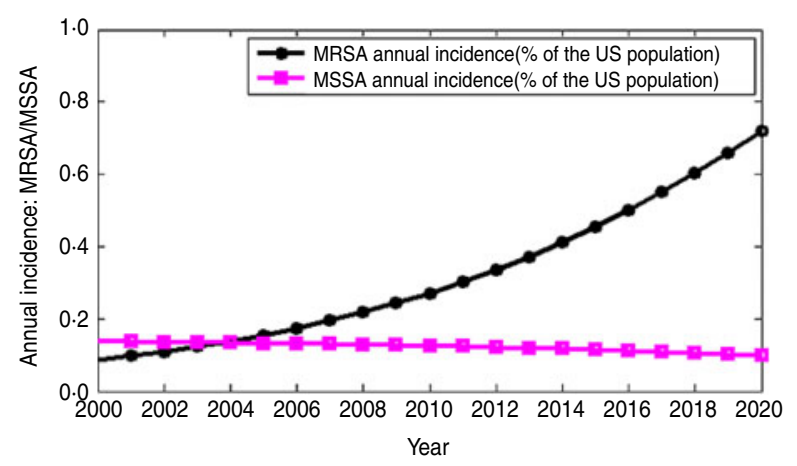

Fig. 3 [colour online]. Baseline model projections: potential MRSA infection incidence increase and gradual replacement of MSSA over the next decade in the USA in the absence of systematic infection control, based on the 1999-2005 national trends (infection and colonization) and assuming strong competition between MRSA and MSSA for colonization of susceptible hosts [19]. (a) Projection of US population growth for period 2000-2020, based on historical US Census data. (b) Model-projected annual incidence of MRSA and MSSA infection, respectively, relative to the US population, over time (years 20002020).

parameters in this order: colonized MSSA clearance rate $\left(\alpha_{2}\right.$, model parameter no. 10 in Table 1), followed closely by the colonized MRSA clearance rate $\left(\alpha_{1}\right.$, model parameter no. 9 in Table 1$)$. This appears the most influential parameter pair, followed by the rate of colonized MSSA progressing to infection $\left(\gamma_{2}\right.$, model parameter no. 12 in Table 1). The fourth and fifth equally influential parameters closely following are then the rate of colonization by colonized MSSA $\left(\lambda_{2}^{\mathrm{C}}\right.$, model parameter no. 5 in Table 1$)$ and the rate of colonized MRSA progressing to infection $\left(\gamma_{1}\right.$, model parameter no. 11 in Table 1), respectively. Coming relatively closely after is the rate of colonization by colonized MRSA $\left(\lambda_{1}^{\mathrm{C}}\right.$, model parameter number 1 in Table 1). Finally, coming last is the pair represented by the rate of infection by colonized MSSA $\left(\beta_{2}^{\mathrm{C}}\right.$, model parameter no. 7 in Table 1) and the rate of infection by colonized MRSA $\left(\beta_{1}^{\mathrm{C}}\right.$, model
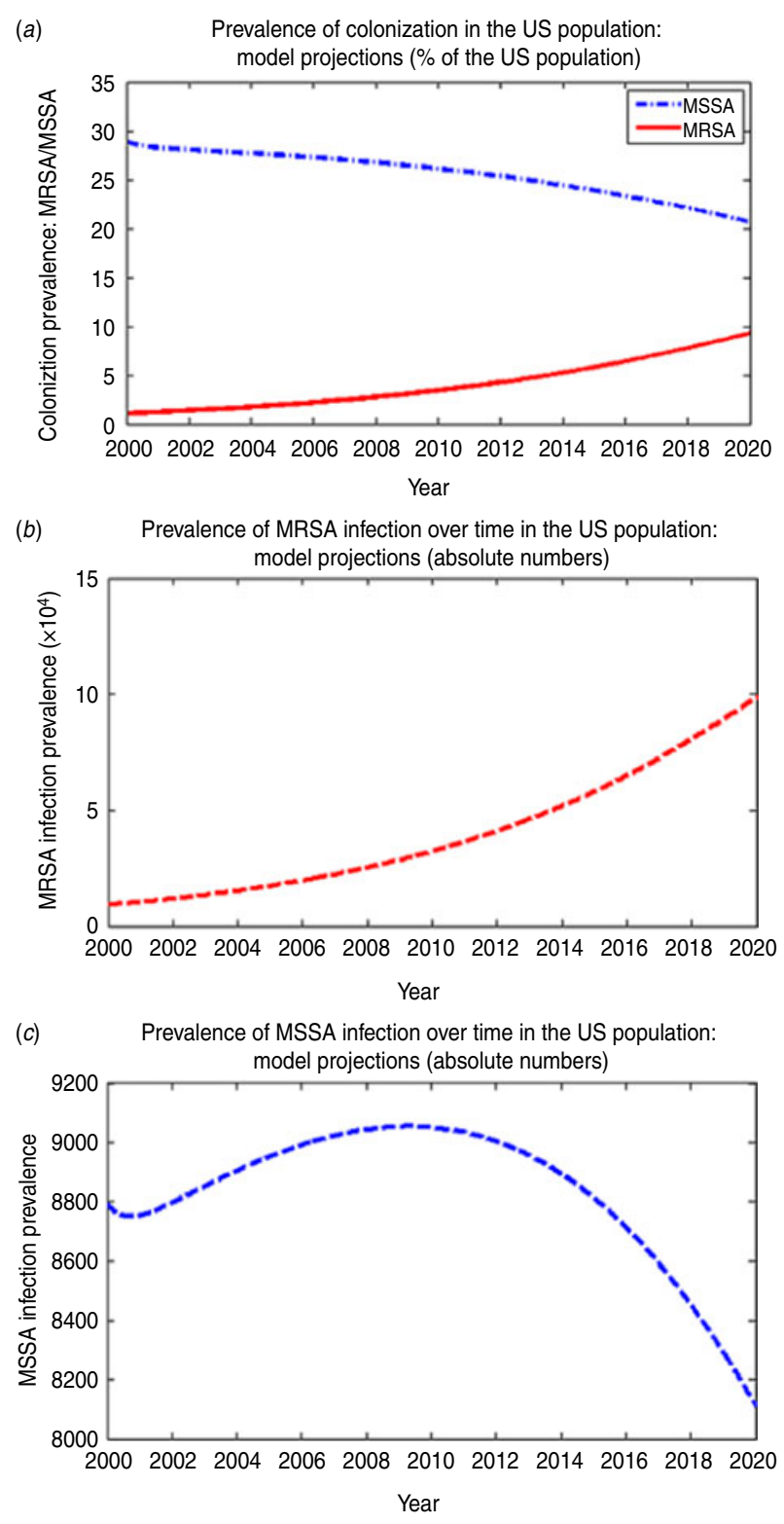

Fig. 4 [colour online]. Baseline model projections for prevalence of (a) MRSA/MSSA colonization, (b) MRSA infection, (c) MSSA infection. Potential MRSA expansion and gradual replacement of MSSA over the next decade in the USA in the absence of systematic infection control, based on the 1999-2005 national trends (infection and colonization) and assuming strong competition between MRSA and MSSA for colonization of susceptible hosts [9]. (a) Model-projected prevalence of MRSA and MSSA colonization, respectively, in the US population over time. (b) Model-projected prevalence of MRSA infection in the US population over time. (c) Model-projected prevalence of MSSA infection in the US population over time.

parameter no. 3 in Table 1), respectively; however, these last two parameters appear notably less influential compared to the previous other six model parameters listed above. The model is significantly less 
(a)

(a) Annual incidence of $S$. aureus infections in the USA (relative to the growing US population)

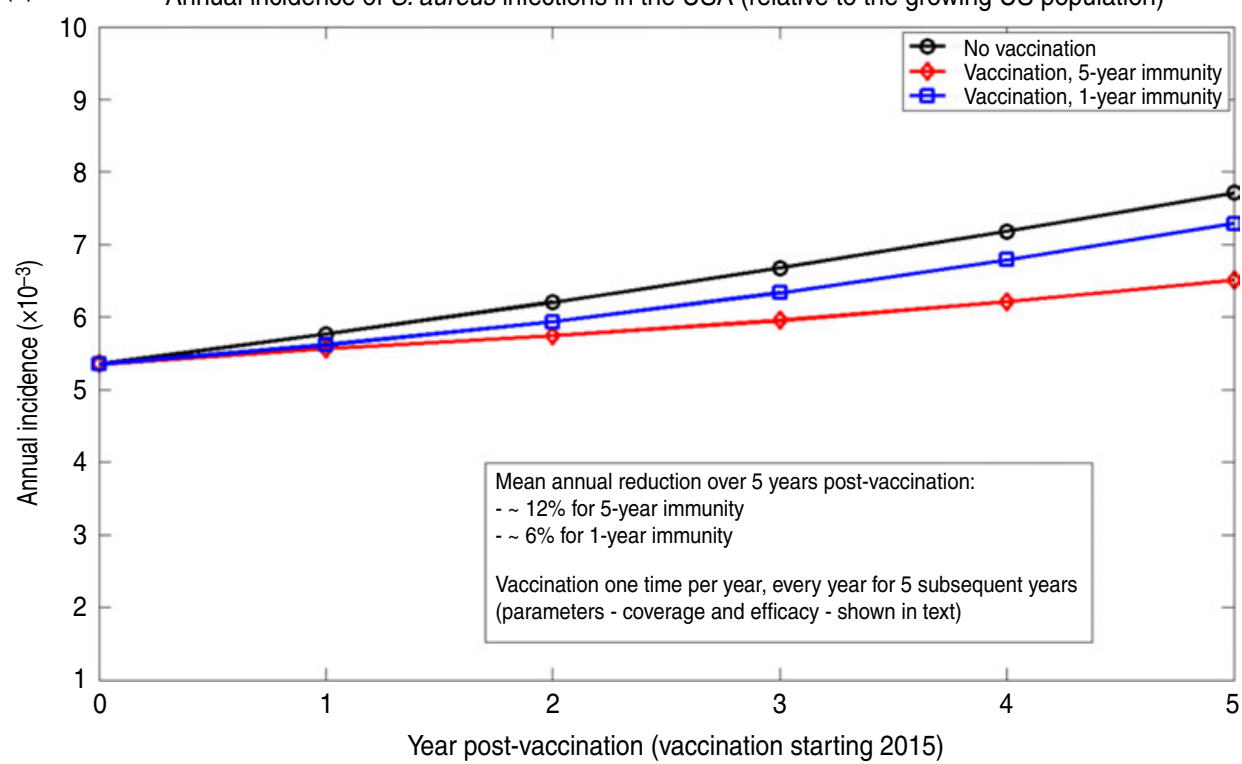

(b) Prevalence of $S$. aureus infection over time in the US population (absolute numbers)

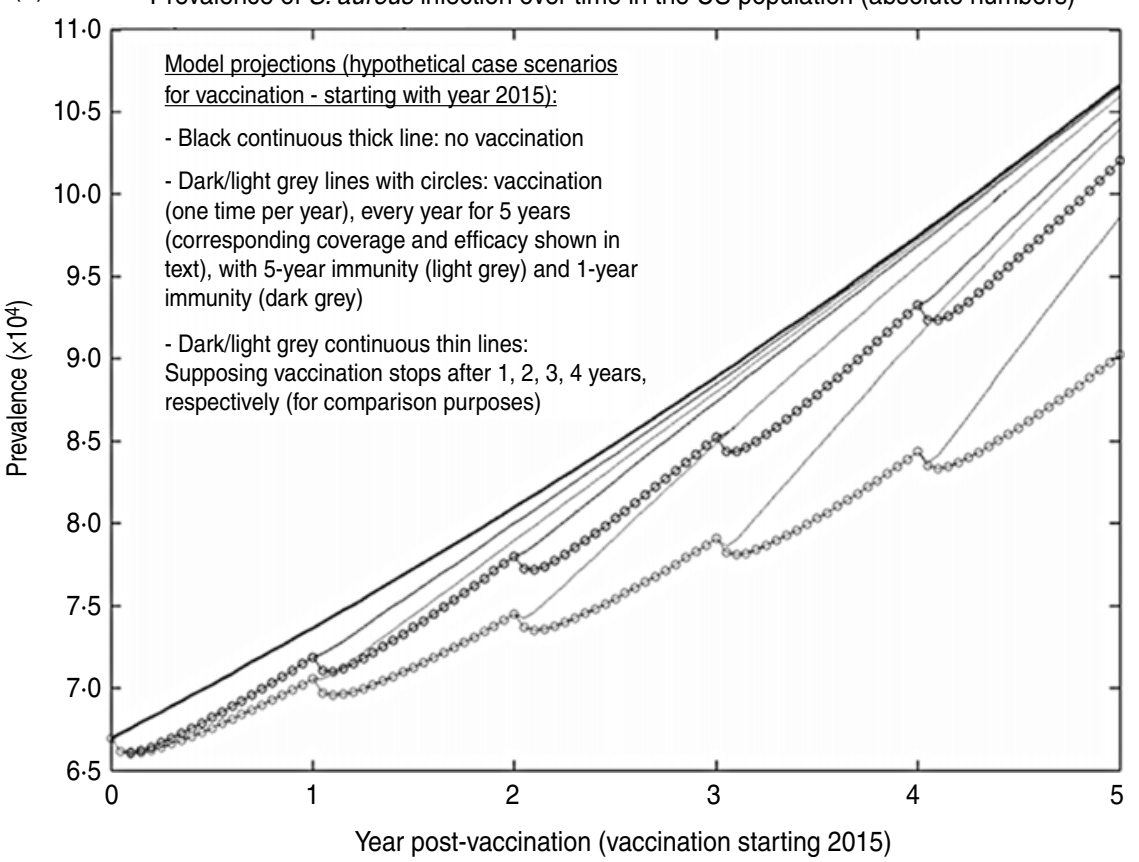

Fig. 5 [colour online]. A model-based hypothetical S. aureus vaccination case scenario. Model projections for (a) incidence and $(b)$ prevalence shown here correspond to annual vaccine coverage of about $5 \%$, with vaccination once per year ('flu-like') and assumed vaccine modes of action (Fig. 1) as follows: $40 \%$ reduction in the risk colonization, 50\% reduction in the risk of direct infection [16] and 40\% reduction in the risk of infection in MRSA/MSSA carriers. (a) Model-projected annual incidence of $S$. aureus infection in the USA (shown here as proportion of the growing population) before and after a hypothetical $S$. aureus vaccination. (b) Model-projected prevalence of $S$. aureus infection in the US population over time (shown here in absolute numbers) before and after a hypothetical $S$. aureus vaccination.

sensitive to the remaining nine model parameters listed in Table 1.

Projected effects of vaccination on $S$. aureus incidence and prevalence over the first 5 years are depicted in Figure 5(a,b), respectively. Under the assumption of a mean duration of vaccine protection of 5 years in vaccinated individuals, the estimated mean annual reduction of $S$. aureus incidence over 
the first 5 years was about $12 \%$. In this case, a reduction of about $16 \%$ was projected for the 5 th (and last) year of this vaccination scenario. However, assuming a mean duration of vaccine protection of only 1 year, the benefits were less marked, with a mean annual reduction of incidence close to $6 \%$ over the first 5 years. The corresponding reductions in prevalence are illustrated in Figure $5 b$, which also highlights the impact of potentially stopping annual vaccination after each subsequent year considered in this simulation (1, 2, 3, 4 years, respectively).

\section{DISCUSSION}

This basic dynamic transmission model for $S$. aureus in the US population explicitly accounts for both MRSA and MSSA. The model was simultaneously calibrated and validated against published US national colonization and infection data, and demonstrated good overall correlation with these data. To our knowledge, this is the first attempt to develop a dynamic transmission model with competition between MRSA and MSSA and calibration/validation against nationally representative colonization/infection data in the US population.

Based on the natural history of infection and nationally representative 1999-2005 data, projections from a simple dynamic transmission model at the US population level suggest how MRSA might continue to expand and gradually replace MSSA over time, assuming competition for colonization. This has already been observed with penicillin, where more than $80 \%$ of the $S$. aureus strains were reported penicillin-resistant by the 1970 s and the community rates increased soon after nosocomial rates exceeded 40-50\% [6]. The model-based estimate of $R_{0}$ emphasizes the need for efficient means of control to limit the spread of infection. Projections for the MSSA growth rate in the near future appear comparable to the estimated US population growth rate, suggesting that the slow growth still seen in projected MSSA infection numbers could potentially be a direct consequence of population growth.

As a secondary objective, we built an exploratory vaccination component on top of our baseline model and illustrated its application here with a hypothetical case scenario, highlighting the potential impact of vaccination on $S$. aureus infection at the US population level. Our results are in line with those published by Lucero et al. [20] who used a population-based surveillance programme to explore the effect of a hypothetical vaccine in preventing invasive MRSA infections. They estimated that vaccination of all adults aged $>65$ years would reduce the incidence of MRSA infections by $12 \cdot 1 \%$ over 1 year. A dynamic transmission modelling framework is versatile and allows for testing and simulation of multiple potential mechanisms of action of a vaccine and the subsequent impact on reducing transmission and spread in a population, as previously demonstrated for $N$. meningitidis [17] or Str. pneumoniae [18].

One of the primary usages of mathematical models of this type is to test and generate/re-generate hypotheses and what-if case scenarios. Here, we set out to investigate the hypothesis of strong MRSAMSSA competition for colonization, which has been proposed by others, and assess corresponding outcomes.

Dall'Antonia et al. [9] concluded that MSSA and MRSA do compete for colonization of the anterior nares (of 680 patients only $0 \cdot 6 \%$ carried both MRSA and MSSA) and estimated a protective efficacy of $78 \%$ for MSSA against colonization with MRSA. This was supported by Lasseter et al. [21] who enrolled 748 subjects across 51 different care homes and failed to find co-colonization of both MRSA and MSSA. Further, Margolis et al. [10], concluded from a rat model of colonization that unlike Haemophilus influenzae or Str. pneumoniae, where multiple strains can co-exist, $S$. aureus strains failed to colonize a host animal with other $S$. aureus present. Other supportive data [22] showed that only $3 \%$ of subjects colonized at least once with $S$. aureus, harboured mixed phenotypes of MSSA and MRSA. The reader is directed to a comprehensive literature review [23] exploring the issue of potential MRSA/MSSA replacement and related competition which concluded that colonization with CA-MRSA may at least partially replace colonization with MSSA.

Potential MSSA/MRSA co-colonization was therefore not explicitly included in this basic model, which could constitute a limitation of the current study. This might have led to an overestimation of the strength of the competition between MRSA and MSSA, with subsequent overestimation of the magnitude of replacement. If further robust nationally representative data become available over longer time-spans that indicate that replacement has not occurred or has been less extensive, then one possible conclusion based on this basic modelling exercise is that MRSA/MSSA competition for colonization is not as strong as speculated in some studies, and that there 
may be more opportunity for co-colonization. In that event, further studies/data would be necessary, particularly to disambiguate $S$. aureus colonization data; the assumption of strong MRSA/MSSA competition for colonization could be further relaxed and included in the model to allow for an explicit niche of co-existence for both MRSA and MSSA [8]. Other limitations of the current study are that no explicit distinction is made in the model between persistent and intermittent colonization or between hospital and community strains, and no age stratification or other heterogeneities of the US population are explicitly considered.

More complex models with increased level of detail would be very interesting and desirable. However, realistically, for practical purposes, model complexity needs to be backed by available data, with corresponding stratification. In the absence of nationally representative data at the US population level with appropriate USA100 vs. USA300 explicit strain stratification, for instance, both in terms of colonization and symptomatic infection, we treated MRSA as a single entity. All resulting parameters, including the corresponding $R_{0}$ value are implicitly understood as averages across all the potential MRSA strains that might be pooled in the data. The model is able to reproduce the data and is focused here on the big picture, in this case interplay between MRSA and MSSA. Strain heterogeneity and genetic diversity over time are beyond the scope of the model. However, models of this type have been developed for several infectious agents with high strain diversity, polyclonality, etc. e.g. Str. pneumoniae, N. meningitidis - and level of complexity depends on the driving questions and available data.

Another useful level of granularity would be to explicitly introduce high-risk $v s$. low-risk populations in the model, but again, in order for such an exercise to be realistic, corresponding data need first to be generated. Technically, from the stand-point of the model structure/complexity, further risk stratification of the population would mean, at a minimum, doubling the number of existing compartments (each of the boxes currently in the model schematic would be split into high-risk and low-risk compartments, respectively), and the introduction of corresponding transmission paths, various transmission parameters and progression rates. This level of complexity is unfortunately not sustainable currently by the data available for calibration. It is not the computational complexity, but the large number of a priori unknown parameters that need to be calibrated based on available data that creates issues.

The same comment stands true for age stratification. For that, all calibration data would need to be age-stratified and the number of model parameters increased. For this reason one must first assess the primary working hypotheses (e.g. strong MRSA/MSSA competition for colonization) and subsequent impact on the outcomes in simpler more basic models such as that presented here.

The data employed here for this basic model calibration, although nationally representative, has its own limitations. NHANES biennial colonization data were publicly available only for the periods 2001-2002 and 2003-2004; the infection data were based on related hospital discharges only; and colonization and infection datasets were for the 1999-2005 time-window only, which is a particular timeframe for $S$. aureus evolution and dynamics in the USA, characterized by the emergence and spread in the community. This may consequently impact the estimated model parameter values and model-based projections. Further additional data points over longer time-spans may help confirm an actual sustained trend $v s$. a transient early phenomenon, and model re-calibration/re-assessment can and should be done in the presence of new evidence and/or data.

Recent publications have suggested that the incidence of invasive healthcare-associated MRSA infections may be decreasing. This is most likely due to the implementation of prevention measures: hand hygiene, physical isolation of patients with infection, environmental decontamination and decolonization of colonized individuals [24]. While the impact of these measures on $S$. aureus at the overall US population level is not known, our dynamic transmission model can be recalibrated when new data become available.

In conclusion, dynamic transmission models are powerful mathematical tools that can help project potential future trends as well as evaluate the potential impact of future population-level interventions. With the proliferation of several antibiotic-resistant bacteria, these models are likely to continue to play an increasingly important role in understanding the transmission of emerging infectious diseases.

\section{SUPPLEMENTARY MATERIAL}

For supplementary material accompanying this paper visit http://dx.doi.org/10.1017/S0950268813001106. 


\section{ACKNOWLEDGEMENTS}

The authors acknowledge Adrian Cassidy and Heather Amrine-Madsen (GlaxoSmithKline Vaccines) for their contribution to this work. We also thank Adriana Rusu (XPE Pharma \& Science on behalf of GlaxoSmithKline Vaccines) who provided writing services, Denis Sohy (GlaxoSmithKline Vaccines), Myriam Willbaux and Wouter Houthoofd (XPE Pharma \& Science on behalf of GlaxoSmithKline Vaccines) for publication coordination and management. GlaxoSmithKline Biologicals SA was the funding source and supported all costs associated with the development and the publishing of the manuscript. All authors had full access to the data and agreed with the submission of the publication.

\section{DECLARATION OF INTEREST}

Cosmina Hogea and Thierry Van Effelterre are employed by the GlaxoSmithKline group of companies (GSK), Camilo J. Acosta was employed by GSK at the time the analysis was performed and is now employed by Merck. Camilo J. Acosta and Thierry Van Effelterre own stock options in GSK. Camilo J. Acosta owns stock options in Merck.

\section{REFERENCES}

1. Hryniewicz W. Epidemiology of MRSA. Infection 1999; 27 (Suppl. 2): S13-S16.

2. Miller LG, Diep BA. Clinical practice: colonization, fomites, and virulence: rethinking the pathogenesis of community-associated methicillin-resistant Staphylococcus aureus infection. Clinical Infectious Diseases 2008; 46: 752-760.

3. Carleton HA, et al. Community-adapted methicillinresistant Staphylococcus aureus (MRSA): population dynamics of an expanding community reservoir of MRSA. Journal of Infectious Diseases 2004; 190: 1730-1738.

4. National Nosocomial Infections Surveillance System. National Nosocomial Infections Surveillance (NNIS) System Report, data summary from January 1992 through June 2004, issued October 2004. American Journal of Infection Control 2004; 32: 470-485.

5. King MD, et al. Emergence of community-acquired methicillin-resistant Staphylococcus aureus USA 300 clone as the predominant cause of skin and soft-tissue infections. Annals of Internal Medicine 2006; 144: 309-317.

6. Chambers HF. The changing epidemiology of Staphylococcus aureus? Emerging Infectious Diseases 2001; 7: 178-182.

7. Klein E, Smith DL, Laxminarayan R. Hospitalizations and deaths caused by methicillin-resistant Staphylo- coccus aureus, United States, 1999-2005. Emerging Infectious Diseases 2007; 13: 1840-1846.

8. Lipsitch M, et al. No coexistence for free: neutral null models for multistrain pathogens. Epidemics 2009; 1: 2-13.

9. Dall'Antonia M, et al. Competition between methicillinsensitive and -resistant Staphylococcus aureus in the anterior nares. Journal of Hospital Infection 2005; 61: 62-67.

10. Margolis E, Yates A, Levin BR. The ecology of nasal colonization of Streptococcus pneumoniae, Haemophilus influenzae and Staphylococcus aureus: the role of competition and interactions with host's immune response. BMC Microbiology 2010; 10: 59.

11. Political Calculations Blogspot (http://politicalcalculations. blogspot.com/2005/11/estimating-us-population.html). Accessed April 2012.

12. Gorwitz RJ, et al. Changes in the prevalence of nasal colonization with Staphylococcus aureus in the United States, 2001-2004. Journal of Infectious Diseases 2008; 197: 1226-1234.

13. Mera RM, et al. Increasing role of Staphylococcus aureus and community-acquired methicillin-resistant Staphylococcus aureus infections in the United States: a 10-year trend of replacement and expansion. Microbial Drug Resistance 2011; 17: 321-328.

14. D'Agata EM, et al. Modeling the invasion of community-acquired methicillin-resistant Staphylococcus aureus into hospitals. Clinical Infectious Diseases 2009; 48: 274-284.

15. Kajita E, et al. Modelling an outbreak of an emerging pathogen. Nature Reviews Microbiology 2007; 5: 700 709.

16. Shinefield H, et al. Use of a Staphylococcus aureus conjugate vaccine in patients receiving hemodialysis. New England Journal of Medicine 2002; 346: 491496.

17. Trotter CL, et al. Optimising the use of conjugate vaccines to prevent disease caused by Haemophilus influenzae type b, Neisseria meningitidis and Streptococcus pneumoniae. Vaccine 2008; 26: 4434-4445.

18. Van Effelterre T, et al. A dynamic model of pneumococcal infection in the United States: Implications for prevention through vaccination. Vaccine 2010; 28: 3650-3660.

19. Diekmann O, Heesterbeek JA, Roberts MG. The construction of next-generation matrices for compartmental epidemic models. Journal of the Royal Society Interface 2010; 7: 873-885.

20. Lucero CA, et al. Evaluating the potential public health impact of a Staphylococcus aureus vaccine through use of population-based surveillance for invasive methicillin-resistant $S$. aureus disease in the United States. Vaccine 2009; 27: 5061-5068.

21. Lasseter G, et al. Staphylococcus aureus carriage in care homes: identification of risk factors, including the role of dementia. Epidemiology and Infection 2010; 138: 686-696.

22. Lee YL, et al. Surveillance of colonization and infection with Staphylococcus aureus susceptible or 
resistant to methicillin in a community skilled-nursing facility. American Journal of Infection Control 1997; 25: 312-321.

23. Mostofsky E, Lipsitch M, Regev-Yochay G. Is methicillin-resistant Staphylococcus aureus replacing methicillin-susceptible $S$. aureus? Journal of Antimicrobial Chemotherapy 2011; 66: 2199-2214.

24. Kallen AJ, et al. Health care-associated invasive MRSA infections, 2005-2008. Journal of the American Medical Association 2010; 304: 641-648. 